ISBN 978-981-11-7861-0

Proceedings of 2018 the 8th International Workshop on Computer Science and Engineering

(WCSE 2018)

Bangkok, 28-30 June, 2018, pp.718722

doi: $10.18178 /$ wcse.2018.06.118

\title{
A Fast Train Wake-up Positioning Method Based on Location Memory
}

\author{
Shunding $\mathrm{Hu}^{3}$, Xiaofei $\mathrm{Gao}^{3}$, Dandan $\mathrm{Liu}^{1,2}$ and Xiangxian $\mathrm{Chen}^{1+}$ \\ ${ }^{1 .}$ College of Biomedical Engineering \& Instrument Science, Zhejiang University, Hangzhou 310027, China \\ ${ }^{2}$ ZZhejiangZheda Train Intelligent Engineering Technology Research Center Co., Ltd, Hangzhou 310051, \\ China \\ ${ }^{3}$ United Science \&Technology Co., Ltd., Research and Development Center, Hangzhou 310051, China
}

\begin{abstract}
This paper mainly studies a fast positioning method of unmanned signal system devices in urban rail transit after dormancy and wake-up of the train, in which layout of corresponding position detection devices in train dormancy wake-up zone is proposed to memory and store the train location information before dormancy. After wake-up of the train, the positioning information obtained through the position detection devices is compared with the positioning information memorized and stored before the dormancy. If the safety verification of the above information is passed, then fast positioning of the train is completed.
\end{abstract}

Keywords: urban rail transit, unmanned, signal system, position memory, train positioning

\section{Introduction}

In the existing CBTC control system of rail transit, before the train is put into operation, it is necessary for the train to be manually powered up and self-checked. Then the train is driven manually in RM mode and runs at low speed in RM mode. After passing through two location beacons in succession, the train obtains effective position information and enters positioning status. The CBTC control system allows the train to enter the driving mode with carbone ATP protection function, such as AM/CM mode, only when the train has the ability to locate safely. The driver is required to participate in the whole process, which takes a long time and the operation efficiency is low.

Under the condition of unmanned driving, no driver is involved to complete the auxiliary train positioning, and the unmanned system needs to complete safety positioning immediately after the train is static or operates autonomously in a small area. Based on this requirement of the unmanned system, this paper proposes a fast and safe positioning method based on location memory for dormancy train after wake-up, which is highly effective as well. According to the different characteristics of the location detection devices placed in the dormancy wake-up zone, there are two fast and safe positioning methods:

\section{ASafe and Fast PositioningMethod Based on Location Memory and Continuous Positioning Devices}

This method requires layout of continuous positioning devices in dormancy wake-up zone of the train, such as positioning loop lines. Layout of continuous positioning devices need to meet requirements of accurate stopping during the train enter into the dormancy wake-up zone, and requirements of fast positioning after wake-up at the front and tail of the train.

In order to meet the requirements of accurate parking for the train to dormancy wake-up zone,the length of the continuous positioning devices for the train parking location should solve the ATP parking error and the reading delay of the continuous positioning devices. The distance $\mathrm{L}$ that from the last read information

Corresponding author. Tel.: + 86-571-87953941;fax: +86-571-87953941.

E-mail address: xxchen99@zju.edu.cn. 
through continuous positioning device to the continuous positioning's reading device when the train stop should satisfy the following formula:

$$
(\mathrm{T} 1+\mathrm{T} 2) * \mathrm{~V} / 3.6<L \leq \frac{\mathrm{D}}{\mathrm{W}}
$$

T1: continuous positioning device's read delay.T2: on-board ATP processing time. V: the allowable speed of the train to dormancy wake-up zone (unit: $\mathrm{km} / \mathrm{h}$ ).D: ATP parking error. W: wheel diameter calibration error. L:the continuous positioning device must be arranged before $\mathrm{L}$ distance to achieve precise parking.

In order to meet the requirements of fast positioning after train waking up, the continuous positioning device's length should be at least to cover the continuous positioning's reading device at the front and end of the train after stopping steady and the distance required for a single jump, the distance L should satisfy the formula:

$$
\mathrm{L} \geq \mathrm{D}+\mathrm{S} \text {. }
$$

D: a single jump distance. S: continuous positioning device's installation error and read side lobe. L: the distance that where the continuous positioning device must be arranged to realize the train location after awakening.

Normally, in order to facilitate the installation and maintenance of the continuous positioning device, the continuous positioning device can be arranged in a way that is full of the whole dormancy wake-up zone.

The continuous positioning devices are shown in figure 1 .

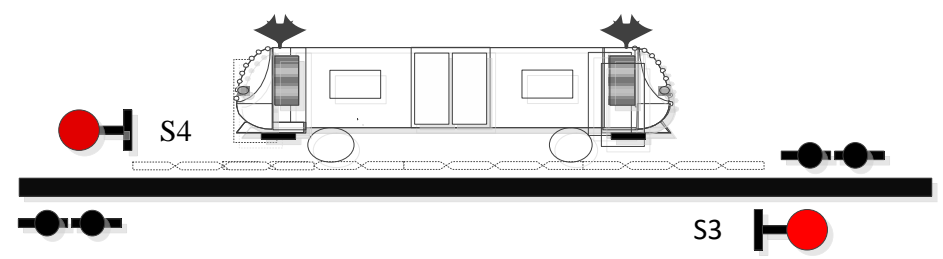

Continuous locating device

Fig. 1: Layout diagram of continuous positioning devices

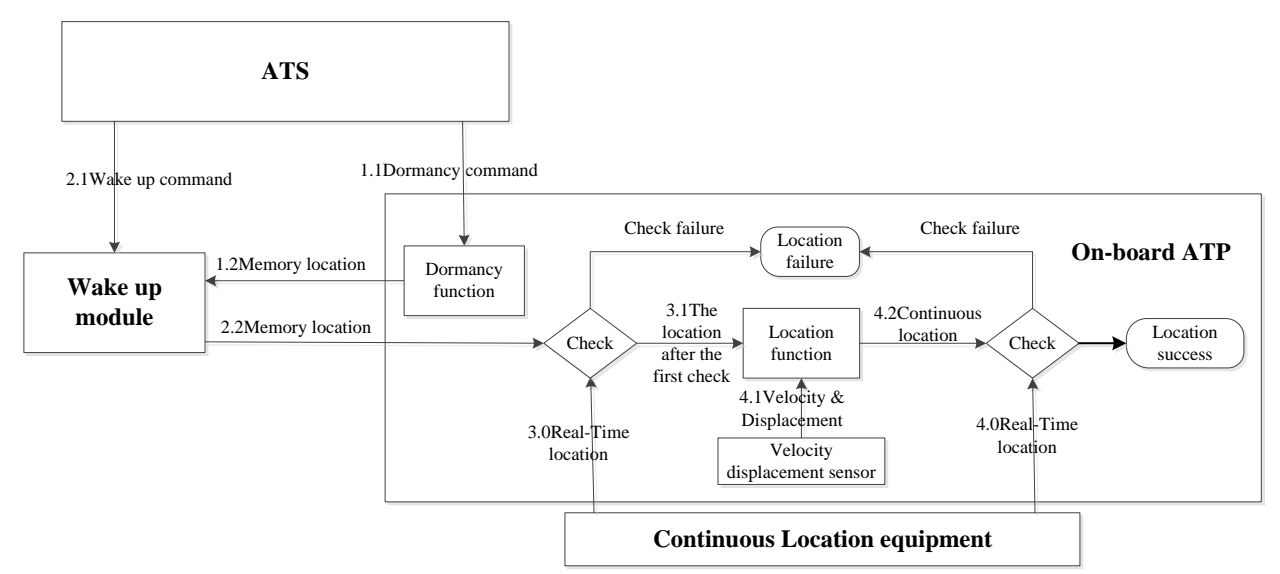

Fig. 2: Flow diagram of safe and fast positioning method based on location memory and continuous location devices

Based on the layout diagram of the continuous positioning devices shown in figure 1, the positioning process of fast train dormancy wake-up based on position memory is as follows:

1) At the end of operation the day before, the train completely entered the wake-up zone and stopped steady and accurately. The carbone controller executed the dormancy process after receiving the dormancy instruction, and stored the location information before the dormancy in its own dormancy wake-up module without failure. 
2) Before starting operation the next day, according to the timetable, the center ATS automatically or manually sends the wake-up instruction to the wake-up module of the carbone controller to wake up the train. After the train wakes up successfully and the carbone controller is powered on, the position information is retrieved from the dormancy wake-up module.

3) The carbone controller verifies the position information obtained from the dormancy wake-up module as well as the safety position information continuously obtained from the positioning devices. If the verification is inconsistent, then failure of the train positioning is determined; while if the verification is successful, then the next train position verification shall be carried out.

4) The carbone controller controls the train to jump in a small range, and the train obtains the position information from the continuous positioning devices during the jump motion. The above obtained position information was compared with the position information obtained for the first time after wake-up and the position information calculated by the velocity displacement sensor, so as to further ensure safety of the train position.

\section{ASafe and Fast PositioningMethod based on Location Memory, Trackside Secondary Occupation and Traditional Positioning Devices}

Compared with method 1, this method does not require layout of continuous positioning devices, and the corresponding transponders are only placed in the dormancy wake-up area. However, in order to realize fast and safe positioning of dormancy wake-up based on traditional positioning devices, system requirements for this method will be higher. Figure 3 shows the specific layout diagram of trackside dormancy wake-up transponders:

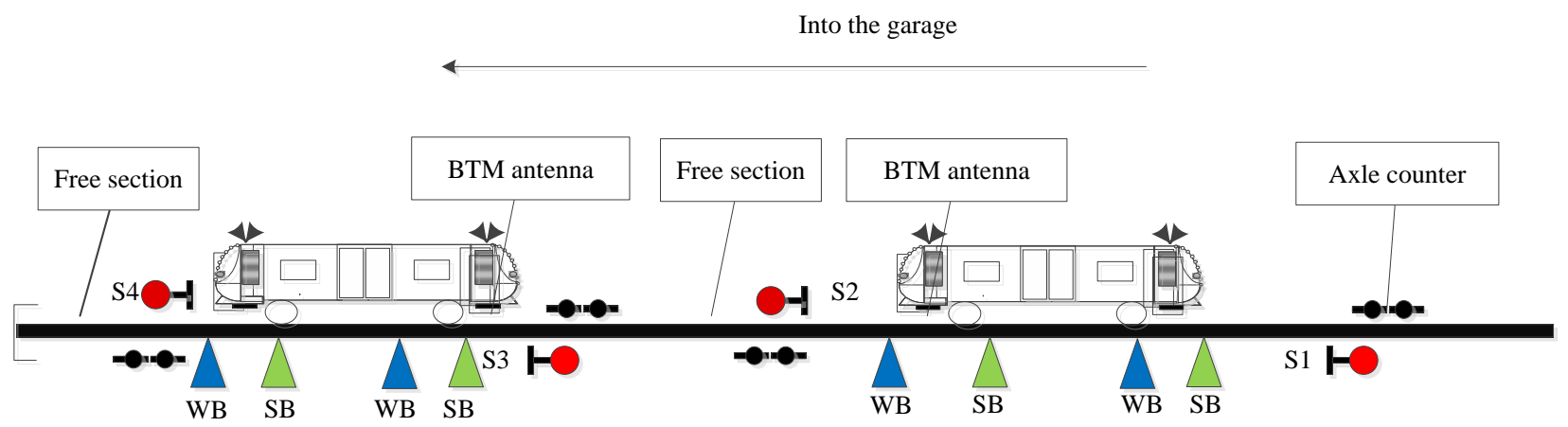

WB Wake up the location transponder

SB Dormancy calibration transponder

Fig. 3: Layout diagram of dormancy wake-up transponders

The above figure shows layout diagram of trackside dormancy wake-up transponders, and the corresponding layout requirements are as follows:

1. It is necessary to reserve a free section between two trains, the train and the train stop, which is used as a safe reserved distance for the electrified jump of the train.

2. In order to ensure the train stops in the preset dormancy wake-up window, position calibration transponders need to be placed in the dormancy wake-up zone to calibrate the position of the train before dormancy.

3. It is necessary to set up a dormancy wake-up window in the wake-up zone to make sure that the train stops in the parking window area steady. Moreover, the dormancy wake-up transponders can be read within the preset jump distance on the premise that the electrified jump on the train does not exceed the preset safe reserved distance.

4. In order to complete the safe re-positioning function of the train, it is necessary to set up dormancy wake-up transponders in the dormancy wake-up zone, which can be used to obtain the location information of the dormancy wake-up transponders through jumping and moving of the train after wake-up. 
Based on the layout diagram of the trackside dormancy wake-up transponders shown in figure 3, the safe and fast positioning process of the train after dormancy wake-up is as follows:

1) At the end of operation the day before, the train completely entered the dormancy wake-up zone, calibrated the position of the train before dormancy according to the position calibration transponders, and stopped in the preset dormancy wake-up parking window steady.

2) According to the timetable, the center ATS automatically or manually sends the dormancy instruction to carbone controller, which executes the dormancy process after receiving the dormancy instruction, and stores the train position information before dormancy in trackside ATP for memory.

3) After the train dormancy is successful, the trackside ATP continuously monitors whether the train has not been moved out of the dormancy wake-up zone according to the secondary train occupation test devices.

4) Before starting operation the next day, according to the timetable, the center ATS automatically or manually sends the wake-up instruction to the wake-up module of the carbone controller to wake up the train. After the train wakes up successfully and the carbone controller is powered on, the position information is retrieved from the trackside ATP, which returns the corresponding location information stored before dormancy to the carbone controller according to the position track of the train during the dormancy.

5) After obtaining the position information stored before the dormancy from the trackside ATP, the carbone controller controls the train to jump in a small area. In the process of jumping, the train obtains the position information from the preset dormancy wake-up positioning transponders, determines the train position with this information, and completes safety positioning of the train.

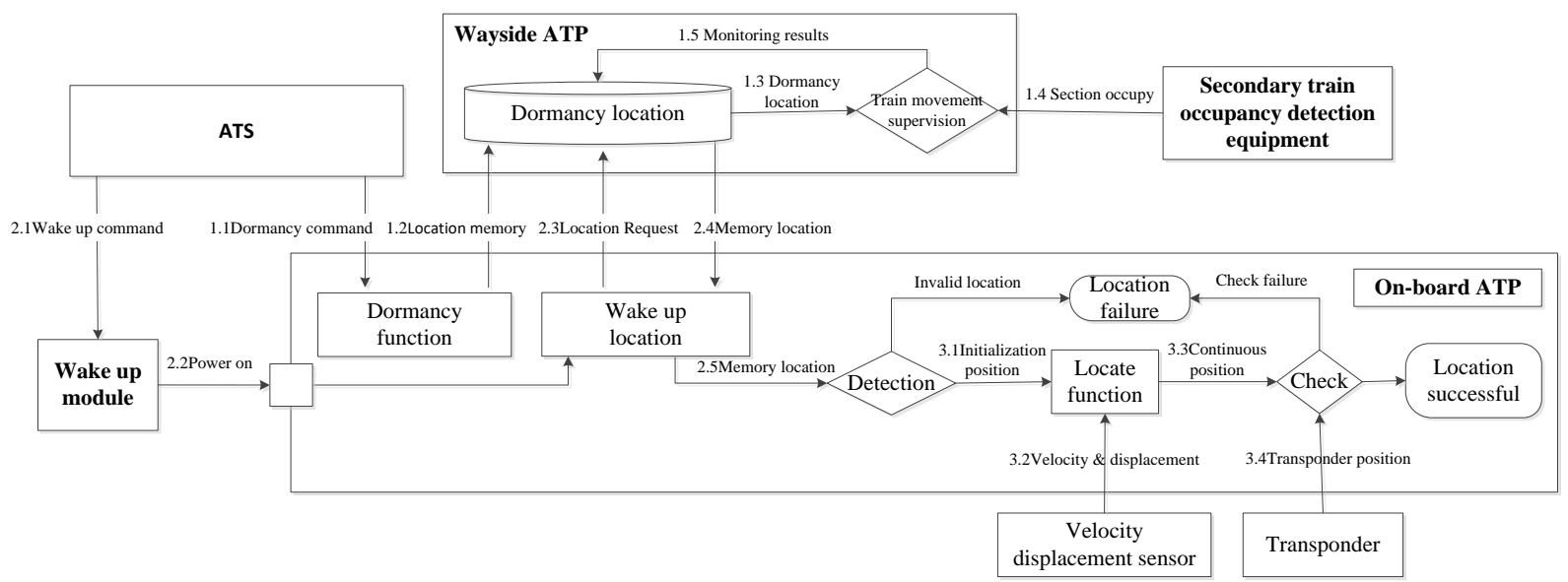

Fig. 4: Flow diagram of a safe and fast location method based on location memory and trackside secondary occupation supervision

\section{Conclusion}

With the wide application of the automatic unmanned signal system, the train positioning technology of the existing signal system is no longer meet the needs of the train positioning after wake-up of the unmanned signal system. This paper can help to solve the problem by offering two safe and fast positioning methods for train fast wake-up based on memory storage.

The first method adopts carbone ATP active positioning technology. First, this method is no longer completely dependent on the location storage and verification results of the trackside ATP, which avoids the situation that the loss of train position in all jurisdiction areas caused by the trackside ATP fault will prevent the train from completing the fast and safe positioning. Second, when there is no jumping interface between the train and the signal system, it can also realize fast positioning of the train, which has a wide range of application. Third, loop line layout of the location detection devices of this method is convenient, which can enter the positioning state before the train is put into operation and improve the operation efficiency.

The second method adopts trackside ATP auxiliary positioning technology. This method is based on the existing hardware devices of traditional CBTC system, and can obtain the safe location information after the unmanned system is powered up and quickly complete the positioning of the train at the minimum cost. 


\section{Acknowledgement}

This work is supported by the funding from the National Science and Technology Infrastructure Program of China under Grant 2015BAG19B03

This work is supported by the funding from National Science and Technology support Plan of China under Grant 2015BAG13B01

\section{References}

[1] AnpingRen. The Development of Fully Automated and Driverless Subway in China.Science\& Technology Vision, 2012(25):207-208.

[2] Yuefan Wang. AUDS--a Brand -new Mode for Urban Mass Transit. Urban Mass Transit,2006(08):1-5.

[3] Xuewei Tian, XiaojuanLIu.Train Position Technique in FAO of Rail Transit. Urban Mass Transit,2017(12):51-54.

[4] Erchao Chen. Discussion on the Engineering Design of Unmanned signal system. Railway Signalling\& Communication Engineering 2017(1):33-36.

[5] Changhai Wu. Discussion on the Application of automatic Unmanned Technology in Urban track. 2016(5):54-58. 